\title{
DESEMPENHO ZOOTÉCNICO E RENDIMENTO DE FILÉ DO ROBALO- FLECHA ALIMENTADO COM DIFERENTES DIETAS COMERCIAIS
}

\author{
Growth performance and fillet of yield common \\ snook fed with different commercial diets \\ Luiz Henrique Castro David ${ }^{1}$, Sara Mello Pinho², Daniel Correia ${ }^{3}$, Mônica Yumi Tsuzuki ${ }^{4}$, \\ Maurício Gustavo Coelho Emerenciano ${ }^{5}$, Giovanni Lemos de Mello ${ }^{6}$

\footnotetext{
${ }^{1}$ Doutorando no Programa de Pós-Graduação em Aquicultura, Centro de Aquicultura da Unesp (Caunesp/Unesp), Jaboticabal, SP, Brasil. Bolsista Capes. E-mail: luiz.h@outlook.com

${ }^{2}$ Doutoranda no Programa de Pós-Graduação em Aquicultura, Centro de Aquicultura da Unesp (Caunesp/Unesp), Jaboticabal, SP, Brasil. Bolsista CNPq. E-mail: sara.pinhoo@hotmail.com

${ }^{3}$ Acadêmico do curso de Engenharia de Pesca, Universidade do Estado de Santa Catarina (Udesc), Laguna, SC, Brasil. E-mail: danielsc-lagu@hotmail.com

${ }^{4}$ Professora do Programa de Pós-Graduação em Aquicultura (PPG-AQI/UFSC), Laboratório de Peixes e Ornamentais Marinhos (Lapom), Universidade Federal de Santa Catarina (UFSC), Florianópolis, SC, Brasil. E-mail: monica.tsuzuki@ufsc.br

${ }^{5}$ Professor do Programa de Pós-Graduação em Zootecnia (PPGZOO/Udesc), Universidade do Estado de Santa Catarina, campus Chapecó, SC, Brasil. CSIRO Agricultura e Alimentação, Programa de Aquicultura, Centro de Pesquisas da Ilha de Bribie, Woorim, QLD, Austrália. E-mail: mauricioemerenciano@hotmail.com ${ }^{6}$ Professor do curso de Engenharia de Pesca, Universidade do Estado de Santa Catarina (Udesc), Laguna, SC, Brasil. E-mail: giovannidemello@gmail.com
}

\begin{abstract}
RESUMO
O objetivo do trabalho foi avaliar o efeito de diferentes dietas comerciais no desempenho zootécnico, nos índices organossomáticos e no rendimento de carcaça e filé do robalo-flecha. Diferentes rações comerciais para peixes carnívoros foram comparadas: Ração A (46,6\% PB e 9,8\% EE) e Ração B (60,6\% PB e 13,3\% EE), na engorda do robalo-flecha. Foram estocados juvenis de robalo-flecha com peso inicial de $355,40 \pm 52,30 \mathrm{~g}$, à densidade de 15 peixes $\mathrm{m}^{-3}\left(5,33 \pm 0,12 \mathrm{~kg} \mathrm{~m}^{-3}\right)$, sob salinidade de $15 \mathrm{~g} \mathrm{~L}^{-1}$, em três tanques de $200 \mathrm{~L}$ com três réplicas. Os tratamentos foram compostos por dois sistemas de recirculação de água independentes. Ao final do experimento, observaram-se diferenças significativas em todos os parâmetros zootécnicos analisados, exceto comprimento padrão, com melhores resultados para peixes alimentados com a Ração B. Para essa ração, os peixes atingiram peso médio final de 501,10 $\pm 64,10 \mathrm{~g}$, proporcionando o dobro de taxa de crescimento e melhor conversão alimentar aparente $(1,17 \pm 0,13)$, entretanto apresentaram maiores valores de gordura visceral e índice hepatossomático em relação à Ração A. Os rendimentos de car-
\end{abstract}

Recebido em: 28/10/2019

Aprovado em: 21/02/2019

Publicado online em: $1^{\circ} . / 11 / 2019$ 
caça e filé analisados não apresentaram diferenças significativas entre os tratamentos. Os resultados indicam que, nessas condições experimentais, a ração contendo $60,6 \%$ PB e $13,3 \%$ EE garante melhor desempenho zootécnico do robalo-flecha cultivado durante 42 dias na fase de engorda.

Palavras-chave: piscicultura marinha, Centropomus undecimalis, nutrição, engorda.

\section{ABSTRACT}

The objective of this work was to evaluate the effect of different commercial diets on the common snook performance, organo-somatic indices and carcass and fillet yield. Two different commercial diets for carnivorous fish were compared in the grow-out stage of the fishes: Feed A (46.6\% CP and $9.8 \%$ fat $)$ and Feed B (60.6\% CP and $13.3 \%$ fat). Common snook juveniles with initial body weight mean of $355.40 \pm 52,30 \mathrm{~g}$ were stocked in three tanks of $200 \mathrm{~L}$ at 15 fish $\mathrm{m}^{-3}$ stocking density $\left(5.33 \pm 0.12 \mathrm{~kg} \mathrm{~m}^{-3}\right)$, at salinity of $15 \mathrm{~g} \mathrm{~L}^{-1}$ with three replicates. The treatments were composed by two independent water recirculation systems. At the end of the experiment, there were significant differences in all performance parameters analyzed, except standard length, with better results for fish fed with Feed B. For this feed, the fish reached an average weight of $501.10 \pm 64.10 \mathrm{~g}$, providing twice the growth rate and better feed conversion $(1.17 \pm 0.13)$ but showed higher values of visceral fat and liver somatic index compared to Feed A. The carcass yield and fillet analyzed showed no significant differences between treatments. The results indicate that under these experimental conditions the feed containing $60.6 \% \mathrm{CP}$ and 13.3 fat ensures better growth performance of common snook grown for 42 days in the grow-out phase.

Keywords: marine fish farming, Centropomus undecimalis, nutrition, grow-out phase.

\section{INTRODUÇÃO}

Na última década, houve um aumento expressivo no interesse de cultivar peixes marinhos em muitos países da América Latina. Isso se deu devido ao potencial zootécnico de várias espécies de alto valor comercial que ocorrem na região e também às condições climático-ambientais e socioeconômicas adequadas para o desenvolvimento da aquicultura (Cerqueira \& Tsuzuki, 2009). Entretanto, a produção desses peixes em tanques-rede ou viveiros estuarinos ainda é pequena e diversos esforços estão sendo conduzidos visando ao desenvolvimento de tecnologias de cultivo, com foco em espécies nativas, especialmente de clima tropical (García-Ortega, 2009).

No Brasil, a piscicultura marinha ainda é incipiente, limitando-se à pesquisa e a poucas iniciativas comerciais, principalmente com o robalo-peva e robalo-flecha (Centropomus parallelus e Centropomus undecimalis), linguado (Paralichthys orbignyanus) e, mais recentemente, com o beijupirá (Rachycentron canadum) (Hamilton et al., 2013; Pinho et al., 2016). Entre essas espécies, o robalo-flecha é reconhecido como um dos peixes marinhos com maior potencial para a aquicultura (Cavalli \& Hamilton, 2009; Passini et al., 2018), visto que possui uma série de características zootécnicas favoráveis, como o rápido crescimento, a alta eficiência nas taxas de conversão alimentar e baixa utilização de energia, além do potencial de ser cultivado em sistemas intensivos, com capacidade de suportar altas biomassas em berçários e na fase de engorda (Alvarez-Lajonchère \& Tsuzuki, 2008; Ibarra- 
Castro et al., 2011). Somado a isso, essa espécie é considerada um recurso de alto valor comercial, sendo também procurada por pescadores esportivos, movimentando um importante segmento do turismo pesqueiro (Sanches et al., 2013; Passini et al., 2018).

No entanto, para que o cultivo do robalo-flecha se torne uma realidade na aquicultura brasileira, algumas práticas devem ser desenvolvidas e/ou melhoradas. Os estudos mais atuais estão focados nos aspectos reprodutivos da espécie (Ibarra-Castro et al., 2011; Rhody et al., 2015; Cruz-Botto et al., 2018; Passini et al., 2018) e poucos têm o objetivo de melhorar o manejo alimentar, mesmo sendo esse um fator importante e um dos principais entraves na piscicultura marinha comercial devido ao seu custo (Barbosa et al., 2011). A alimentação pode representar mais de 50\% dos custos de produção (Rana et al., 2009) e poucas marcas comerciais disponibilizam rações específicas para as diferentes espécies marinhas. Aliado a isso, o correto manejo alimentar é fundamental para alcançar taxas ideais de crescimento e atender aos demais índices zootécnicos de interesse produtivo (ex. rendimento de filé), podendo auxiliar também na manutenção do estado de saúde dos peixes em confinamento (Mello et al., 2015), o que reforça a necessidade de mais estudos nessa área.

As exigências nutricionais dos robalos ainda não foram totalmente determinadas e as informações obtidas até o momento são insuficientes para se estabelecer dietas adequadas (Costa-Filho et al., 2013). Por causa disso, em situações práticas de cultivo normalmente são fornecidas rações de outras espécies carnívoras, por apresentarem semelhanças com o hábito alimentar dos robalos (Tsuzuki \& Berestinas, 2008). No entanto, poucos trabalhos foram conduzidos utilizando e comparando dietas disponíveis no mercado para a fase de engorda do robalo-flecha (Alvarez-Lajonchère \& Tsuzuki, 2008). Diante dessa carência de informações científicas a respeito da nutrição do robalo-flecha, importante pilar para o desenvolvimento da criação em escala produtiva, e cientes de que estudos sobre o processamento de novas espécies dão subsídios à indústria e indicam importante característica sobre o seu potencial para o mercado de pescado, o objetivo deste trabalho foi avaliar o efeito de diferentes dietas comerciais no desempenho zootécnico, nos índices organosomáticos e no rendimento do filé de robalo-flecha na fase de engorda.

\section{MATERIAL E MÉTODOS}

\section{Delineamento experimental}

O experimento foi realizado no Laboratório de Aquicultura (LAQ), da Universidade do Estado de Santa Catarina (Udesc), Laguna, SC, durante 42 dias. Juvenis de robalo-flecha foram previamente mantidos em aclimatação a $25^{\circ} \mathrm{C}$, salinidade de $15 \mathrm{~g} \mathrm{~L}^{-1}$ e alimentados com ração comercial para peixes carnívoros, com $45 \%$ de proteína bruta (PB).

Os peixes foram estocados nas unidades experimentais à densidade de 15 peixes $\mathrm{m}^{-3}$ $\left(5,33 \pm 0,12 \mathrm{~kg} \mathrm{~m}^{-3}\right)$, com peso médio de 355,40 \pm 52,30 g, comprimento total de 36,60 \pm 1,70 cm e comprimento padrão de 30,30 $\pm 1,60 \mathrm{~cm}$. Durante 42 dias, foram avaliados dois tratamentos, em triplicata, diferenciados entre si pela utilização de duas rações comerciais distintas (Tabela I): Ração A, formulada para peixes carnívoros de água doce, e Ração B, utilizada na larvicultura de peixes marinhos em laboratório. Para isso, dois sistemas independentes e idênticos de recirculação de água foram utilizados, cada um com três tanques de $200 \mathrm{~L}$ conectados a um macrocosmo de $150 \mathrm{~L}$. Em ambos os macrocosmos havia filtros mecânico e biológico, carvão ativado, bomba para o retorno da água (Atman®, $\mathrm{PH}$ 3500 ) e termostato-aquecedores (Atman $\left.{ }^{\circledR}, 300 \mathrm{~W}\right)$, como descrito por Mello et al. (2015). 
Tabela I - Composição das dietas comerciais em base úmida, utilizadas na engorda final de juvenis de robalo-flecha, Centropomus undecimalis, em sistema de recirculação de água

\begin{tabular}{lrr}
\multicolumn{1}{c}{$\begin{array}{c}\text { Composição } \\
\text { (g/100 g de matéria seca) }\end{array}$} & Ração A & Ração B \\
\hline Proteína bruta & 46,62 & 60,59 \\
Extrato etéreo & 9,79 & 13,33 \\
Carboidratos & 15,25 & 0,00 \\
Fibra digerida em ácido (FDA) & 8,07 & 3,78 \\
Cinzas & 11,70 & 15,43 \\
Umidade & 8,57 & 6,91 \\
Energia total (kJ.g-1) & 4,52 & 4,83 \\
Proteína/energia (mg proteína.kJ-1 $)$ & 103,90 & 125,36 \\
Energia proteica/energia não proteica & 1,40 & 2,43 \\
\hline
\end{tabular}

Em relação ao processamento das dietas comerciais, ambas passaram por processo de repeletização para padronizar o tamanho dos pellets. Para isso, foram moídas, peneiradas para uniformização dos grânulos e submetidas à adição do aglutinante diluído (gelatina sem sabor + água), na proporção de $10 \mathrm{~g}$ de aglutinante para cada quilo de ração (1\% do peso de ração moída). O aglutinante foi diluído em $400 \mathrm{ml}$ de água quente e aos poucos adicionado à ração moída até adquirir consistência. A mistura final foi então repeletizada (8 $\mathrm{mm}$ de diâmetro) e seca em estufa por 24 horas, a $50^{\circ} \mathrm{C}$.

Os peixes foram alimentados até a saciedade aparente duas vezes ao dia (às 9h e 17h). A ração não consumida foi removida do tanque, com auxílio de um puçá, minutos após o término do manejo alimentar. Os resíduos alimentares e fezes foram sifonados diariamente. A quantidade de ração oferecida foi pesada e calculada descontando os pellets não consumidos, obtendo uma boa estimativa da quantidade de ração consumida pelos peixes.

Os valores de proteína bruta, extrato etéreo, fibras, cinzas e umidade foram determinados por meio de análises bromatológicas realizadas de acordo com a metodologia proposta pela Association of Official Analitycal Chemists (AOAC, 1999). Os valores de carboidratos foram estimados pela diferença entre o total de nutrientes na ração $(100 \%)$ e a soma dos teores de proteína, lipídios, fibras e cinzas analisada (Gracia-López et al., 2006). A energia bruta foi calculada com valores médios de energia de combustão dos nutrientes $\left(5,6 \mathrm{kcal} \mathrm{g}^{-1}\right.$ de proteína bruta, 9,4 kcal g-1 de lipídeos e 4,1 $\mathrm{kcal} \mathrm{g}^{-1}$ de fibra e carboidrato) (Maynard \& Loosli, 1974).

\section{Parâmetros de qualidade da água}

Durante o experimento, os níveis de oxigênio dissolvido foram mantidos próximo à saturação por meio de um sistema de aeração artificial. $\mathrm{O}$ fotoperíodo foi de $12 \mathrm{~h}$ Luz : $12 \mathrm{~h}$ Escuro. Diariamente, pela manhã e à tarde, às $10 \mathrm{~h}$ e $18 \mathrm{~h}$ respectivamente, eram monitorados a temperatura e o oxigênio dissolvido da água com auxílio de oxímetro polarográfico YSI 55 (Yellow Springs Instrument Company, Yellow Springs, Ohio, USA), salinidade com refratômetro e $\mathrm{pH}$ por meio de pHmetro (EcoSense, YSI). A cada 14 dias foram monitoradas as concentrações de amônia total, nitrito, nitrato, ortofosfato e alcalinidade por meio de kit colorimétrico AT 101 (Alfakit, Florianópolis, SC, Brasil).

\section{Biometrias e índices zootécnicos}

As biometrias foram realizadas no início (dia 0) e no final (dia 42) do experimento, medindo o peso corporal úmido (P) (precisão 0,01 g), comprimento total (CT) e compri- 
mento padrão $(\mathrm{CP})$ (precisão de $0,1 \mathrm{~cm}$ ), submetendo os peixes à anestesia com óleo de cravo (75 $\left.\mathrm{mg} \mathrm{L}^{-1}\right)$ para facilitar o manuseio dos mesmos. Não houve diferenças significativas $(\mathrm{p}>0,05)$ entre os pesos iniciais, $\mathrm{CT}_{\text {inicial }}$ e $\mathrm{CP}_{\text {inicial }}$ nos diferentes tratamentos.

Por meio dos resultados obtidos, foram calculados sobrevivência, ganho de peso (GP), incremento de biomassa (IB), taxa de crescimento específico (TCE) e conversão alimentar aparente $(\mathrm{CA})$, sendo: $\mathrm{K}=$ [peso final $(\mathrm{g}) /$ comprimento final $(\mathrm{cm})]^{3} \times 100 ; \mathrm{GP}=$ peso final (g) - peso inicial (g), por unidade experimental; IB = biomassa final (g) - biomassa inicial $(\mathrm{g})$, por unidade experimental; TCE $=(\ln P f-\ln P i) / T \times 100$, sendo $\ln P f$ : logaritmo natural do peso final; lnPi: logaritmo natural do peso inicial e T: tempo de duração do experimento em dias; $\mathrm{CA}=$ total de ração consumida $(\mathrm{g}) /$ ganho de peso $(\mathrm{g})$.

\section{Análises de rendimento}

Para a realização das análises de rendimento de carcaça, os peixes foram mantidos em caixas térmicas, com gelo, até o momento da filetagem. Foram processados manualmente por um filetador treinado 12 peixes (6 por tratamento), com pesagem das porções obtidas a partir do peixe inteiro (Corrêa et al., 2013). Os valores de rendimento (\%) foram obtidos em relação ao peso total do peixe inteiro, e o peso obtido das amostras após aplicar os seguintes cortes: a) peixe eviscerado e com cabeça (peixe inteiro sem as vísceras, brânquias, escamas e nadadeiras), b) peixe eviscerado sem cabeça e c) filé (musculatura sem ossos e pele, obtida em dois cortes laterais).

\section{Análises dos órgãos internos}

$\mathrm{Na}$ caracterização anatômica do aparelho gastrointestinal foram utilizados os mesmos 12 peixes (6 de cada tratamento), em que foram aplicados os cálculos de rendimento de filé. Os robalos foram submetidos a jejum de dois dias para evitar interferências do alimento nos pesos do estômago e intestino. As medidas de comprimento foram realizadas com auxílio de paquímetro, em milímetros, e os pesos foram medidos por meio de balança digital de precisão, em gramas. Para expressar quanto cada órgão interno e a gordura visceral representam no peso total do peixe, foram calculados os seguintes índices organossomáticos: Índice Hepatossomático (IHS = ((Peso fresco do fígado g)/(Peso corporal g) $\times 100)$; Índice Estomagossomático (IES = ((Peso fresco do estômago g) $/($ Peso corporal g) $) \times 100)$; Índice Intestinossomático (IIS = ((Peso fresco do intestino g) $/($ Peso corporal $)) \times 100)$; Índice Esplenossomático $($ IEP $=(($ Peso fresco do baço g $) /($ Peso corporal $))$ $x 100)$; Relação Comprimento do Intestino/Comprimento Total $=\left(\mathrm{C}_{\text {intestino }} / \mathrm{CT}\right)$; e Gordura Visceral $(\mathrm{GV}=(($ Peso fresco da gordura visceral g) $/($ Peso corporal $)) \times 100)$.

\section{Análise estatística}

A análise estatística foi realizada comparando os dados pelo teste t-Student. A homocedasticidade das variáveis foi verificada pelo teste de Levene e a normalidade pelo teste de Kolmogorov-Smirnov. Para todas as análises foi adotado o nível de significância de 5\%.

\section{RESULTADOS E DISCUSSÃO}

Os parâmetros físico-químicos de qualidade de água mantiveram-se do início ao fim do experimento em níveis adequados à espécie (Cerqueira, 2004) (Tabela II). Não houve 
mortalidade em nenhuma unidade experimental ao longo dos 42 dias de ensaio. Adicionalmente, os peixes cultivados não apresentaram sinal clínico de enfermidade ou qualquer outra anormalidade. Os resultados de desempenho zootécnico são apresentados na Tabela III.

Tabela II - Parâmetros físico-químicos de água nos diferentes tratamentos de engorda final do robalo-flecha

\begin{tabular}{lrr}
\hline Parâmetro & Ração A & \multicolumn{1}{c}{ Ração B } \\
\hline Oxigênio $\left(\mathrm{mg} \mathrm{L}^{-1}\right)$ & $6,90 \pm 0,53^{\mathrm{a}}$ & $6,82 \pm 0,47^{\mathrm{a}}$ \\
Temperatura $\left({ }^{\circ} \mathrm{C}\right)$ & $28,53 \pm 0,99^{\mathrm{a}}$ & $28,59 \pm 1,02^{\mathrm{a}}$ \\
Salinidade $\left(\mathrm{g} \mathrm{L}^{-1}\right)$ & $10,84 \pm 1,33^{\mathrm{a}}$ & $10,66 \pm 1,34^{\mathrm{a}}$ \\
Amônia $\left(\mathrm{mg} \mathrm{L}^{-1}\right)$ & $1,01 \pm 1,20^{\mathrm{a}}$ & $1,27 \pm 1,48^{\mathrm{a}}$ \\
Nitrato $\left(\mathrm{mg} \mathrm{L}^{-1}\right)$ & $0,45 \pm 0,31^{\mathrm{a}}$ & $0,59 \pm 0,28^{\mathrm{a}}$ \\
Nitrito $\left(\mathrm{mg} \mathrm{L}^{-1}\right)$ & $0,05 \pm 0,02^{\mathrm{a}}$ & $0,05 \pm 0,05^{\mathrm{a}}$ \\
Ortofosfato $\left(\mathrm{mg} \mathrm{L}^{-1}\right)$ & $1,82 \pm 0,76^{\mathrm{a}}$ & $2,78 \pm 1,41^{\mathrm{a}}$ \\
Alcalinidade $\left(\mathrm{mg} \mathrm{L}^{-1} \mathrm{de} \mathrm{CaCO}_{3}\right)$ & $21,20 \pm 9,98^{\mathrm{a}}$ & $28,00 \pm 8,64^{\mathrm{a}}$ \\
\hline
\end{tabular}

Nota: valores apresentados como média \pm DP. Letras iguais indicam médias sem diferença significativa entre os tratamentos, pelo teste t-Student a 5\% de significância.

Tabela III - Resultados zootécnicos da engorda do robalo-flecha, Centropomus undecimalis, alimentados com diferentes dietas comerciais durante 42 dias

\begin{tabular}{lrr}
\hline \multicolumn{1}{c}{ Parâmetros zootécnicos } & \multicolumn{1}{c}{ Ração A } & \multicolumn{1}{c}{ Ração B } \\
\hline Peso corporal úmido $(\mathrm{g})$ & $422,20 \pm 50,40^{\mathrm{b}}$ & $501,10 \pm 64,10^{\mathrm{a}}$ \\
Comprimento total $(\mathrm{cm})$ & $37,40 \pm 1,40^{\mathrm{b}}$ & $38,30 \pm 1,70^{\mathrm{a}}$ \\
Comprimento padrão $(\mathrm{cm})$ & $31,39 \pm 1,30^{\mathrm{a}}$ & $32,20 \pm 1,53^{\mathrm{a}}$ \\
Ganho de peso $(\mathrm{g})$ & $66,31 \pm 3,03^{\mathrm{b}}$ & $146,10 \pm 29,64^{\mathrm{a}}$ \\
Incremento de biomassa $(\mathrm{g})$ & $198,93 \pm 9,10^{\mathrm{b}}$ & $438,37 \pm 88,91^{\mathrm{a}}$ \\
Consumo alimentar diário $(\mathrm{g})$ & $7,66 \pm 0,74^{\mathrm{b}}$ & $12,05 \pm 1,27^{\mathrm{a}}$ \\
Consumo alimentar total $(\mathrm{g})$ & $321,61 \pm 31,12^{\mathrm{b}}$ & $506,00 \pm 53,17^{\mathrm{a}}$ \\
Conversão alimentar aparente & $1,62 \pm 0,10^{\mathrm{b}}$ & $1,17 \pm 0,13^{\mathrm{a}}$ \\
\hline
\end{tabular}

Nota: valores apresentados como média \pm DP. Letras iguais indicam médias sem diferença significativa entre os tratamentos. Letras diferentes indicam médias com diferença significativa entre os tratamentos, pelo teste t-Student a 5\% de significância.

Durante o período experimental, juvenis de robalo-flecha com peso médio inicial de

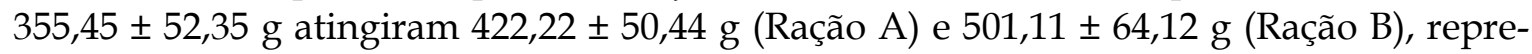
sentando incremento de $18,78 \%$ e $40,98 \%$ respectivamente. O peso final e o ganho de peso foram maiores $(p<0,05)$ no tratamento que utilizou a Ração B, em comparação com os peixes alimentados com a Ração A. A taxa de crescimento específico (TCE) seguiu essa mesma tendência, sendo superior, com valores duas vezes maiores para os peixes alimentados com a Ração B $\left(0,84\right.$ contra $0,37 \%$ dia $\left.^{-1}\right)(p<0,05)$ (Figura 1$)$.

A necessidade de altos níveis de proteína para atender aos processos vitais e proporcionar o crescimento de peixes carnívoros, somado ao elevado custo desse nutriente, confere importância fundamental à determinação da exigência proteica nas dietas (Tucker Jr., 1987). Segundo Cerqueira (2004), no caso dos robalos, é aconselhável que o nível de proteína bruta seja acima de 50\% e o de lipídeos em torno de 9\%. Souza et al. (2011), traba- 
lhando com desempenho zootécnico e econômico de juvenis de robalo-peva (C. parallelus) alimentados com dietas de diferentes concentrações proteicas (37,5\% a $49 \%)$, encontraram relação direta entre o aumento da proteína, o ganho de peso total e a TCE. De acordo com Tucker Jr. (2005), dietas para engorda com 50\%-56\% de proteína (com até $30 \%$ de proteína de origem da soja), 13\%-14\% de lipídeos e 13\%-25\% de carboidratos apresentaram resultados satisfatórios para o cultivo do robalo-flecha.

Oviedo-Pérez et al. (2013) apre-

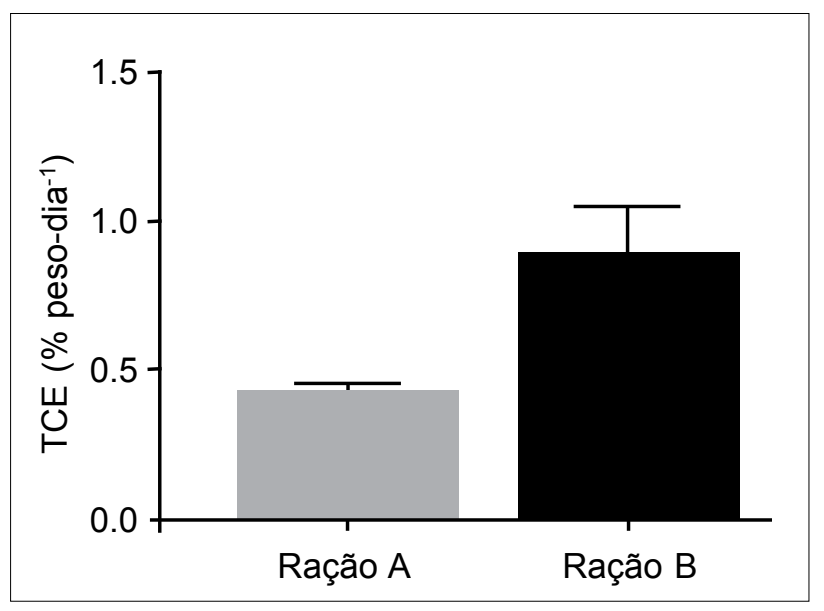

Figura 1 - Taxa de crescimento específico (TCE) para juvenis de robalo-flecha, Centropomus undecimalis, alimentados com duas dietas comerciais durante 42 dias sentaram uma curva de crescimento para o robalo-flecha cultivado durante dois anos em gaiolas, na Laguna del Ostion, Veracruz, México. O estudo avaliou as fases de pré-engorda e engorda, desde 3,3 g até 820 g, utilizando rações com $42 \%$ de proteína e $12 \%$ de lipídeos. Os peixes cresceram de 300 a $820 \mathrm{~g}$ em oito meses, resultando em uma TCE de $0,40 \%$ dia $^{-1}$, similar à TCE de $0,37 \%$ dia $^{-1}$ encontrada no presente estudo para os peixes alimentados com a Ração A. Supondo que a melhoria nas formulações de dietas para o robalo-flecha possa contribuir para o alcance de TCE próxima a $0,80 \%$ dia $^{-1}$ nesta mesma fase (300 a 820 g), conforme obtido com a Ração B, esse período supracitado poderia ser atingido em apenas quatro meses, ou seja, uma redução de $50 \%$ no tempo de cultivo na fase de engorda.

A taxa de conversão alimentar foi significativamente melhor com a Ração B $(1,17)$ em comparação à Ração A $(1,60)$. Em uma simulação de custo de produção, esse acrescimento de $0,43 \mathrm{~kg}$ de ração para cada kg produzido com a Ração A pode representar uma diferença de custos com ração de $\mathrm{R} \$ 17.200,00$, considerando uma produção de 10 toneladas de peixe por ano e o preço da ração de $\mathrm{R} \$ 4,00$ por $\mathrm{kg}$. Um estudo avaliando a influência da densidade de estocagem no cultivo de juvenis de robalo-flecha mantidos em laboratório encontrou taxas de conversão alimentar de 1,88, 2,06 e 2,30, inversamente proporcional à densidade de estocagem empregada (Souza-Filho \& Cerqueira, 2003). Contudo, taxas de conversão alimentar próximas a 1, ou até inferiores, são reportadas por alguns autores para o robalo-flecha (Tucker Jr., 1987).

Vale ressaltar que, ao comparar com o estudo de Souza-Filho e Cerqueira (2003), no cultivo experimental de juvenis de robalo-flecha $(\sim 60 \mathrm{~g})$, alimentados com ração de $52 \%$ de proteína e $10 \%$ de lipídeos, com uma variação da temperatura entre $23,3{ }^{\circ} \mathrm{C}$ e $30,6{ }^{\circ} \mathrm{C}$, os autores apresentaram valores médios de CA e TCE de 2,0\% e 0,7 \% .dia ${ }^{-1}$ respectivamente. Os resultados satisfatórios de CA e TCA do presente estudo podem também ser atribuídos à faixa de temperatura mantida durante todo o experimento, próxima ao conforto térmico da espécie, com valores médios de $28,5 \pm 0,1^{\circ} \mathrm{C}$ (Winner et al., 2010).

Para o comprimento padrão $(\mathrm{CP})$ não foram identificadas diferenças estatísticas nas medidas finais. Esse resultado provavelmente deve-se ao fato de que os robalos, nessa fase, apresentarem maior incremento de peso do que de comprimento. Ximenes-Carvalho (2006), trabalhando com modelos de idade e crescimento de robalos no Sudeste do Brasil, encontrou uma equação potencial para robalo-flecha: $W=0,00000298 \mathrm{~L}^{3,285}$. Como exemplo, 
pela aplicação na fórmula, peixes de $38,28 \mathrm{~cm}$ deveriam ter peso total de $472,37 \mathrm{~g}$, ou seja, dentro da faixa de desvio padrão do comprimento total encontrado no presente estudo $(501,11 \pm 64,12 \mathrm{~g})$.

O melhor desempenho encontrado nos peixes cultivados com a Ração B, para a maioria dos parâmetros zootécnicos avaliados, pode ser atribuído ao alto nível proteico e ao maior consumo da ração. Esses resultados sugerem maior palatabilidade do alimento e corroboram com o estudo de Tsuzuki e Berestinas (2008). Esses autores avaliaram o efeito de diferentes dietas comerciais no crescimento de juvenis de robalo-peva $(4,10 \pm 0,10 \mathrm{~g})$ e visualizaram que a dieta para camarões com $45 \%$ PB e 7\% EE, com alto teor de proteína, era mais palatável e proporcionou o melhor desempenho do robalo-peva.

Foram utilizados níveis proteicos muito diferentes entre as duas rações, os quais foram definidos por meio de análise bromatológica (Tabela I), com 46,6\% PB na Ração A e $60,6 \%$ na Ração B. No entanto, outros fatores, como o perfil de ácidos graxos, de aminoácidos, a qualidade das matérias-primas e o correto balanceamento da relação proteína/ energia, também são importantes e podem ter afetado os resultados. Todos esses já foram elucidados como fatores-chave na nutrição de peixes marinhos (Glencross, 2009; Small \& Soares Jr., 1998; Gao et al., 2016) e são determinantes para o sucesso ou fracasso no cultivo comercial de peixes marinhos (Tacon \& Metian, 2008). Embora os resultados zootécnicos tenham mostrado que é desejável optar por uma ração comercial com características semelhantes à Ração B, estudos voltados para o desenvolvimento de dietas específicas para essa espécie são incentivados.

Quanto ao rendimento de carcaça e filé, não houve diferenças significativas entre os tratamentos (Tabela IV). Os dados apresentaram-se similares a peixes marinhos de carne branca, como truta arco-íris (Oncorhynchus mykiss) (Macedo-Viegas et al., 2002) e beijupirá (Rachycentron canadum) (Gonçalves et al., 2014). O rendimento médio de filé para o robaloflecha obtido no presente estudo foi de $42,5 \%$, similar ao encontrado por Corrêa et al. (2013) em robalos-peva obtidos de ambiente de água doce $(44,5 \%)$ e de ambiente marinho $(43,1 \%)$. Contudo, Tucker Jr. (2003) aponta rendimento de filé de 57\% a 60\% para robalos-flecha pesando entre 350 e $500 \mathrm{~g}$, superior aos valores médios obtidos para barramundi (Lates calcarifer), com $40 \%$, segundo esse mesmo autor. Embora esses rendimentos encontrados possam ser considerados altos, o filé não é o único produto de interesse na cadeia produtiva, já que o pequeno piscicultor, por exemplo, muitas vezes comercializa seu produto inteiro.

Tabela IV - Rendimento de carcaça (peixes eviscerados com cabeça e sem cabeça) e filé do processamento do robalo-flecha

\begin{tabular}{ccccc}
\hline \multirow{2}{*}{ Tratamento } & \multicolumn{4}{c}{ Rendimento (\%) } \\
\cline { 2 - 5 } & Inteiro & $\begin{array}{c}\text { Eviscerado com } \\
\text { cabeça }\end{array}$ & $\begin{array}{c}\text { Eviscerado sem } \\
\text { cabeça }\end{array}$ & Filé \\
\hline Ração A & 100 & $89,8 \pm 0,7^{\mathrm{a}}$ & $78,2 \pm 2,7^{\mathrm{a}}$ & $43,1 \pm 2,0^{\mathrm{a}}$ \\
Ração B & 100 & $91,0 \pm 0,9^{\mathrm{a}}$ & $78,1 \pm 0,9^{\mathrm{a}}$ & $42,0 \pm 1,1^{\mathrm{a}}$ \\
\hline
\end{tabular}

Nota: valores apresentados como média \pm DP. Letras iguais indicam médias sem diferença significativa entre os tratamentos, pelo teste t-Student a 5\% de significância.

Na análise dos índices organossomáticos, não foram encontradas diferenças significativas para a maioria dos índices avaliados, exceto para o índice hepatossomático e o de gordura visceral (Tabela V). Os peixes apresentaram maior índice hepatossomático quando alimentados com a Ração A. Estudos apresentados por Kubitza, Guimarães e Kubitza (2000) dizem que peixes supridos com alimentos ricos em carboidratos e extrato etéreo, 
porém com níveis inferiores de proteína (características que corroboram a formulação da Ração A), tendem a apresentar aumento no tamanho do fígado, podendo prejudicar as funções desse órgão e possivelmente suprimir o crescimento do animal.

\begin{tabular}{ccc}
\multicolumn{3}{l}{$\begin{array}{l}\text { Tabela V - Índices organossomáticos e gordura visceral do robalo-flecha, alimentado com diferentes } \\
\text { dietas comerciais, durante } 42 \text { dias em sistemas de recirculação de água }\end{array}$} \\
\hline \multicolumn{1}{c}{ Índices } & Ração A & Ração B \\
\hline Índice hepatossomático (\%) & $1,01 \pm 0,25^{\mathrm{a}}$ & $0,68 \pm 0,07^{\mathrm{b}}$ \\
Índice estomagossomático (\%) & $0,50 \pm 0,05^{\mathrm{a}}$ & $0,48 \pm 0,07^{\mathrm{a}}$ \\
Índice intestinossomático (\%) & $0,66 \pm 0,06^{\mathrm{a}}$ & $0,57 \pm 0,03^{\mathrm{a}}$ \\
Índice esplenossomático (\%) & $0,05 \pm 0,01^{\mathrm{a}}$ & $0,05 \pm 0,01^{\mathrm{a}}$ \\
Relação cintestino: CT & $0,55 \pm 0,03^{\mathrm{a}}$ & $0,52 \pm 0,05^{\mathrm{a}}$ \\
Gordura visceral (\%) & $0,75 \pm 0,18^{\mathrm{a}}$ & $2,06 \pm 0,34^{\mathrm{b}}$ \\
\hline
\end{tabular}

Nota: valores apresentados como média \pm DP. Letras iguais indicam médias sem diferença significativa entre os tratamentos. Letras diferentes indicam médias com diferença significativa entre os tratamentos, pelo teste t-Student a 5\% de significância.

Os valores mais altos de gordura visceral foram encontrados nos peixes alimentados com a Ração B, fato possivelmente explicado pelo maior teor de extrato etéreo em sua composição. Pinto (2011), em experimento com juvenis de robalo-peva, encontrou a mesma tendência de deposição de gordura na cavidade visceral com o aumento do teor de lipídios e energia nas dietas. Entretanto, Lie (2001) e Glencross (2009) mostraram que, além do consumo de ácidos graxos da dieta, os níveis de gordura visceral também são influenciados pelo correto balanceamento da relação energia-proteína. Fatores esses que precisam ser melhor elucidados em trabalhos futuros com a espécie.

\section{CONCLUSÃO}

A Ração B, contendo 60,6\% PB e 13,3\% EE, proporcionou melhores índices zootécnicos na fase de engorda do robalo-flecha. No entanto, essa ração aumentou o acúmulo de gordura visceral e o índice hepatossomático. Devido a isso, embora o crescimento tenha sido diferente, o rendimento de carcaça e o de filé foram semelhantes entre os peixes cultivados com as duas dietas. Nesse sentido, o presente trabalho mostra que é possível realizar a engorda (350-500 g) de robalo-flecha num período de 42 dias, com melhores resultados produtivos, utilizando uma ração com composição semelhante à Ração B.

Agradecimentos - Ao Conselho Nacional de Desenvolvimento Científico e Tecnológico (CNPq), pelo auxílio financeiro, ao Laboratório de Piscicultura Marinha (LAPMAR-UFSC), pelo fornecimento dos peixes para a realização do experimento, e a Danúbio Aquacultura Ltda.

\section{REFERÊNCIAS BIBLIOGRÁFICAS}

Alvarez-Lajonchère, L. \& Tsuzuki, M.Y. A review of methods for Centropomus spp. (snooks) aquaculture and recommendations for the establishment of their culture in Latin America. Aquac. Res., v. 39, n. 7, p. 684-700, 2008.

AOAC (Association of Official Analytical Chemists). In Cunniff, P. (ed.). Official Methods of Analysis of AOAC. Washington, 1141 p., 1999. 
Barbosa, M.C.; Neves, F.F. \& Cerqueira, V.R. Taxa alimentar no desempenho de juvenis de robalo-peva em tanque-rede. Acta Sci. Anim. Sci., v. 33, n. 4, p. 369-372, 2011.

Cavalli, R.O. \& Hamilton, S. Piscicultura marinha no Brasil com ênfase na produção do beijupirá. Rev. Bras. Rep. Anim. Supl., v. 6, p. 64-69, 2009.

Cerqueira, V.R. Cultivo de peixes marinhos, in Poli, C.R. et al. Aquicultura experiências brasileiras. Multitarefa, p. 369-406, 2004.

Cerqueira, V.R. \& Tsuzuki, M.Y. A review of spawning induction, larviculture, and juvenile rearing of the fat snook, Centropomus parallelus. Fish Phys. Bioch., v. 35, n. 1, p. 17-28, 2009.

Corrêa, C.F.; Tachibana, L.; Leonardo, A.F. \& Baccarin, A.E. Body yield, fillet composition and sensory evaluation of river and sea fat snook. Bol. Inst. Pes., v. 39, n. 4, p. 401-410, 2013.

Costa-Filho, J.; Fabregat, T.E.H.P. \& Rosa, C.A.V.L. Uma revisão sobre os principais aspectos no cultivo do robalo. Rev. Ciên. Agrov., v. 12, n. 3, p. 317-325, 2013.

Cruz-Botto, S.; Roca-Lanao, B.; Gaitán-Ibarra, S.; Chaparro-Muñoz, N. \& Villamizar, N. Natural vs laboratory conditions on the reproductive biology of common snook Centropomus undecimalis (Bloch, 1792). Aquac., v. 482, p. 9-16, 2018.

Gao, Y.J.; Liu, Y.J.; Chen, X.Q.; Yang, H.J. \& Tian, L.X. Total aromatic amino acid requirement of juvenile grass carp (Ctenopharyngodon idella). Aquac. Nut., v. 22, n. 4, p. 865-872, 2016.

García-Ortega, A. Nutrition and feeding research in the spotted rose snapper (Lutjanus guttatus) and bullseye puffer (Sphoeroides annulatus), new species for marine aquaculture. Fish Phys. Bioc., v. 35, n. 1, p. 69-80, 2009.

Glencross, B.D. Exploring the nutritional demand for essential fatty acids by aquaculture species. Rev. Aquac., v.1, p. 71-124, 2009.

Gonçalves, A.A.; Dantas-Neto, A.B.; Guilherme, D.D.; Marques, M.K.; Sales, T.M.O.; Lima, J.T.A.X.; Ribeiro, F.A.S. \& Diógenes, A.F. Rendimento de cortes e qualidade da carne do beijupirá, Rachycentron canadum, sujeito a diferentes gradientes de salinidade da água de cultivo, p. 154-165, in Nunes, A.J.P. (ed.). Ensaios com o beijupirá, Rachycentron canadum: resultados e experiências do Projeto Nutrição, sanidade e valor do beijupirá, Rachycentron canadum, cultivado no Nordeste do Brasil. 352 p., Fortaleza, 2014.

Gracia-López, V.; Rosas-Vázquez, C. \& Brito-Pérez, R. Effects of salinity on physiological conditions in juvenile common snook Centropomus undecimalis. Comparative Biochemistry and Physiology - Mol. Integ. Phys., v. 145, n. 3, p. 340-345, 2006.

Hamilton, S.; Severi, W. \& Cavalli, R.O. Biology and aquaculture of cobia: a review. Bol. Inst. Pesca, v. 39, n. 4, p. 461-477, 2013.

Ibarra-Castro, L.; Alvarez-Lajonchère, L.; Rosas, C.; Palomino-Albarrán, I.G.; Holt, G.J. \& Sanchez-Zamora, A. GnRHa-induced spawning with natural fertilization and pilot-scale juvenile mass production of common snook, Centropomus undecimalis (Bloch, 1792). Aquac., v. 319, n. 3-4, p. 479-483, 2011.

Kubitza, L.M.M.; Guimarães, T.G. \& Kubitza, F. Monitorando a saúde dos peixes. Pan. Aquic., p. 32-39, 2000.

Lie, Ø. Flesh quality - the role of nutrition. Aquac. Res., v. 32, n. 1998, p. 341-348, 2001. 
Macedo-Viegas, E.M.; Souza, M.L.R.; Zuanon, J.A.S. \& Faria, R.H.S. Rendimento e composição centesimal de filés in natura e pré-cozido em truta arco-íris, Oncorhynchus mykiss (Wallbaum). Acta Scient., v. 1, p. 1191-1195, 2002.

Maynard, L. \& Loosli, J.K. Nutrição animal. Freitas Bastos, 550 p., 1974.

Mello, G.L.; Jeronimo, G.T.; Tancredo, K.R.; Brol, J.; Almeida, E.J.; Martins, M.L. \& Tsuzuki, M.Y. Development and health status of Centropomus undecimalis parasitized by Rhabdosynochus rhabdosynochus (Monogenea) under different salinity and temperature conditions. Rev. Bras. Paras. Vet., v. 24, n. 3, p. 350-356, 2015.

Oviedo-Pérez, A.; Sanchez-Zamora, A.; Oviedo-Aceves, A. \& Pineda-Sandoval, C. Centropomus undecimalis obtained in Lab: growth of juvenile floating cages in Veracruz, México, in Anais Book of Abstracts, World Aquaculture Society, 2013. Disponível em: < https:/ / www.was.org/meetings/ShowAbstract.aspx?Id=28760>. Acesso em: 11 out. 2018.

Passini, G.; Sterzelecki, F.C.; Carvalho, C.V.A. de; Baloi, M.F.; Naide, V. \& Cerqueira, V.R. 17a-Methyltestosterone implants accelerate spermatogenesis in common snook, Centropomus undecimalis, during first sexual maturation. Theriog., v. 106, p. 134-140, 2018.

Pinho, S.M.; Brol, J.; Almeida, E.J. de; Mello, G.L. de; Jerônimo, G.T. \& Emerenciano, M.G. C. Effect of stocking density and vertical substrate addition on growth performance and health status of fat snook Centropomus parallelus. Aquac., v. 457, p. 73-78, 2016.

Pinto, R.C.C. Aporte lipídico em dietas de juvenis do robalo, Centropomus parallelus, para um máximo crescimento em cultivos com água estuarina. Dissertação de mestrado, Programa de Pós-Graduação em Ciências Marinhas Tropicais, Universidade Federal do Ceará, 73 p., Fortaleza, 2011.

Rana, K.J.; Siriwardena, S. \& Hasan, M.R. Impact of rising feed ingredient prices on aquafeeds and aquaculture production. FAO Fisheries and Aquaculture Technical Paper, v. 541, 63 p., 2009.

Rhody, N.R.; Davie, A.; Zmora, N.; Zohar, Y.; Main, K.L. \& Migaud, H. Influence of tidal cycles on the endocrine control of reproductive activity in common snook (Centropomus undecimalis). Gen. Comp. End., v. 224, p. 247-259, 2015.

Sanches, E.G.; Mello, G.L. de \& Amaral Junior, H. Primeira ocorrência de malformação na coluna vertebral em juvenis de robalo-flecha. Bol. Inst. Pes., v. 39, n. 1, p. 77-83, 2013.

Small, B.C. \& Soares, J.H. Estimating the quantitative essential amino acid requirements of striped bass Morone saxatilis, using fillet A/E ratios. Aquac. Nut., v. 4, n. 4, 225-232, 1998.

Souza, J.H.; Fracalossi, D.M.; Garcia, A.S.; Ribeiro, F.F. \& Tsuzuki, M.Y. Desempenho zootécnico e econômico de juvenis de robalo-peva alimentados com dietas contendo diferentes concentrações proteicas. Pesq. Agrop. Bras., v. 46, n. 2, p. 190-195, 2011.

Souza-Filho, J.J. \& Cerqueira, V.R. Influência da densidade de estocagem no cultivo de juvenis de robalo-flecha mantidos em laboratório. Pesq. Agrop. Bras., v. 38, n. 11, p. 13171322, 2003.

Tacon, A.G.J. \& Metian, M. Global overview on the use of fish meal and fish oil in industrially compounded aquafeeds: trends and future prospects. Aquac., v. 285, n. 1-4, p. 146-158, 2008.

Tsuzuki, M.Y. \& Berestinas, A.C. Performance of juveniles of the fat snook Centropomus parallelus at different. Bol. Inst. Pes., v. 34, n. 4, p. 535-541, 2008. 
Tucker Jr., J.W. Snook and tarpon snook culture and preliminary evaluation for commercial farming. Prog. Fish-Cult., v. 49, p. 49-57, 1987.

Tucker Jr., J.W. Snook culture. Wo. Aqua. Mag., v. 34, n. 4, p. 42-46, 2003.

Tucker Jr., J.W. Snook culture. Amer. Fish. Soc. Symp., v. 46, p. 297-305, 2005.

Winner, B.L.; Blewett, D.A.; McMichael, R.H. \& Guenther, C.B. Relative abundance and distribution of common snook along shoreline habitats of Florida Estuaries. Trans. Amer. Fish. Soc., v. 139, p. 62-79, 2010.

Ximenes-Carvalho, M.O. Idade e crescimento do robalo-flecha, Centropomus undecimalis (Bloch, 1792) e robalo-peva, Centropomus parallelus (Poey, 1860) (Osteichthyes: Centromidae), no Sudeste do Brasil. Dissertação de mestrado, Programa de Pós-Graduação em Ciências Marinhas Tropicais, Universidade Federal do Ceará, 90 p., Fortaleza, 2006. 